\title{
Degree of Approximation of Conjugate of Signals (Functions) by Lower Triangular Matrix Operator
}

\author{
Vishnu Narayan Mishra ${ }^{1}$, Huzoor H. Khan ${ }^{2}$, Kejal Khatri ${ }^{1}$ \\ ${ }^{1}$ Department of Mathematics, S. V. National Institute of Technology, Surat, India \\ ${ }^{2}$ Department of Mathematics, Aligarh Muslim University, Aligarh, India \\ E-mail:vishnu_narayanmishra@yahoo.co.in,huzoorkhan@yahoo.com,kejal0909@gmail.com \\ Received May 4, 2011; revised October 25, 2011; accepted November 5, 2011
}

\section{Abstract}

In the present paper, an attempt is made to obtain the degree of approximation of conjugate of functions (signals) belonging to the generalized weighted $W\left(L_{P}, \xi(t)\right),(p \geq 1)$-class, by using lower triangular matrix operator of conjugate series of its Fourier series.

Keywords: Conjugate Fourier Series, Generalized Weighted $W\left(L_{P}, \xi(t)\right)$-Class, Degree of Approximation and Lower Triangular Matrix Means

\section{Introduction}

Let $f$ be a $2 \pi$-periodic signal (function) and let $f \in L_{1}[0,2 \pi]=L_{1}$. Then the Fourier series of a function (signal) $\mathrm{f}$ at any point $x$ is given by

$$
\begin{aligned}
f(x) & \approx \frac{a_{0}}{2}+\sum_{k=1}^{\infty}\left(a_{k} \cos k x+b_{k} \sin k x\right) \\
& \equiv \sum_{k=0}^{\infty} u_{k}(f ; x),
\end{aligned}
$$

with partial sums $s_{n}(f ; x)$ - a trigonometric polynomial of degree (or order) $n$, of the first $(n+1)$ terms.

The conjugate series of Fourier series (1.1) of $f$ is given by

$$
\sum_{k=1}^{\infty}\left(b_{k} \cos k x-a_{k} \sin k x\right) \equiv \sum_{k=1}^{\infty} v_{k}(f ; x)
$$

with partial sums $\tilde{s}_{n}(f ; x)$.

If $f$ is Lebesgue integrable and $p \geq 1, f \in \operatorname{Lip}(\xi(t), p)$, then

$$
2 \pi \tilde{f}(x)=-\int_{0}^{\pi} \psi(t) \cot (t / 2) \mathrm{d} t=-\lim _{h \rightarrow 0} \int_{h}^{\pi} \psi(t) \cot (t / 2) \mathrm{d} t,
$$

exists for all $x$ Zygmund [1, p. 131], $\tilde{f}(x)$ is called the conjugate function of $f(x)$.

The matrix $\mathrm{T} \equiv\left(a_{n, k}\right)$, in which $a_{n, k}$ is the element in $n$-th row and $k$-th column is usually called the matrix of $\mathrm{T}$. Matrices $\mathrm{T}$ such that $a_{n, k}=0$, for $k>n$, are called lower triangular.
Let $\mathrm{T} \equiv\left(a_{n, k}\right)$ be an infinite lower triangular matrix satisfying Töeplitz [2] conditions of regularity, i.e.

$\sum_{k=0}^{n}\left|a_{n, k}\right| \leq M$, where $M$ is a finite constant independent of $n$,

$\lim _{n \rightarrow \infty} a_{n, k}=0$, for each $k>n$ and $\lim _{n \rightarrow \infty} \sum_{k=0}^{n} a_{n, k}=1$.

Let $\sum_{n=0}^{\infty} u_{n}$ be an infinite series whose $(k+1)^{\text {th }}$ partial sum $s_{k}=\sum_{n=0}^{k} u_{n}$.

The sequence-to-sequence transformation

$$
\tau_{n}=\sum_{k=0}^{\infty} a_{n, k} s_{k}=\sum_{k=0}^{\infty} a_{n, n-k} s_{n-k}, n=0,1,2, \cdots,
$$

defines the sequence $\left\{\tau_{n}\right\}$ of lower triangular matrix summability means of sequence $\left\{s_{n}\right\}$ generated by the sequence of coefficients $\left(a_{n, k}\right)$. The transforms $\tau_{n}$ are called linear means or matrix means (determined by the matrix T) of the sequence $\left\{s_{n}\right\}$.

An infinite series $\Sigma u_{n}$ is said to be summable to s by lower triangular matrix T-method, if $\lim _{n \rightarrow \infty} \tau_{n}$ exists and is equal to s Zygmund [1, p. 74] and we write $\tau_{n} \rightarrow s(T)$, as $n \rightarrow \infty$ The summability matrix $\mathrm{T}$ or the sequenceto-sequence transformation $\tau_{n}$ is said to be regular, if $\lim _{n \rightarrow \infty} s_{n}=s \Rightarrow \lim _{n \rightarrow \infty} \tau_{n}=s$.

A function (signal) $f(x) \in \operatorname{Lip} \alpha$, for $0<\alpha \leq 1$, if $|f(x+t)-f(x)|=\mathrm{O}\left(t^{\alpha}\right)$. 
A function (signal) $f(x) \in \operatorname{Lip}(\alpha, p)$ for $p \geq 1,0<\alpha \leq 1$, Fadden [3], if

$$
\left\{\int_{0}^{2 \pi}|f(x+t)-f(x)|^{p} \mathrm{~d} x\right\}^{1 / p}=\mathrm{O}\left(t^{\alpha}\right),
$$

Given a positive increasing function $\xi(t)$ and an integer $p \geq 1, f(x) \in \operatorname{Lip}(\xi(t), p)$, Khan [4], if

$$
\left\{\int_{0}^{2 \pi}|f(x+t)-f(x)|^{p} \mathrm{~d} x\right\}^{1 / p}=\mathrm{O}(\xi(t)) .
$$

In case $\xi(t)=t^{\alpha}, 0<\alpha \leq 1$, then $\operatorname{Lip}(\xi(t), p)$ coincides with the class $\operatorname{Lip}(\alpha, p)$. If $p \rightarrow \infty$ in $\operatorname{Lip}(\alpha, p)$ class then this class reduces to Lip $\alpha$.

For a given positive increasing function $\xi(t)$, an integer $p \geq 1, f(x) \in W\left(L_{p}, \xi(t)\right)$, Khan [4], if

$$
\left\{\int_{0}^{2 \pi}\left|\{f(x+t)-f(x)\} \sin ^{\beta} x\right|^{p} \mathrm{~d} x\right\}^{1 / p}=\mathrm{O}(\xi(t)),(\beta \geq 0) .
$$

We note that, if $\beta=0$ then the generalized weighted $W\left(L_{p}, \xi(t)\right),(p \geq 1)$-class coincide with the class $\operatorname{Lip}(\xi(t), p)$.

Also we observe that

$$
\operatorname{Lip} \alpha \subseteq \operatorname{Lip}(\alpha, p) \subseteq \operatorname{Lip}(\xi(t), p) \subseteq W\left(L_{p}, \xi(t)\right)
$$

for $0<\alpha \leq 1, p \geq 1$, Mishra [5].

The $L_{p}$-norm is defined by

$$
\|f\|_{p}=\left(\int_{0}^{2 \pi}|f(x)|^{p} \mathrm{~d} x\right)^{1 / p}, p \geq 1 .
$$

The $L_{\infty}$-norm of a function $f: R \rightarrow R$ is defined by

$$
\|f\|_{\infty}=\sup \{|f(x)|: x \in R\},
$$

and the degree of approximation $E_{n}(f)$ of a function $f: R \rightarrow R$ is given by

$$
E_{n}(f)=\operatorname{Min}_{n}\left\|f(x)-\tau_{n}(f ; x)\right\|_{p},
$$

in terms of $n$, where $\tau_{n}(f ; x)$ is a trigonometric polynomial of degree (order) $n$. This method of approximation is called trigonometric Fourier Approximation (tfa) Mishra [6]. Riesz-Hölder Inequality states that if $p$ and $q$ be non-negative extended real numbers such that $1 / p+1 / q=1$. If $f \in L^{p}[a, b]$ and $g \in L^{q}[a, b]$, then $f . g \in L^{1}[a, b]$ and

$$
\int_{a}^{b}|f g| \leq\|f\|_{p}\|g\|_{q} .
$$

Equality holds if and only if, for some non-zero constants $A$ and $B$, we have $A|f|^{p}=B|g|^{q}$ a.e.

Second Mean Value theorem for integration states that if $G:[a, b] \rightarrow R$ is a positive monotonically decreasing function and $\phi:[a, b] \rightarrow R$ is an integrable function, then $\exists$ a number $x \in(a, b)$ such that

$$
\int_{a}^{b} G(t) \phi(t) \mathrm{d} t=G(a+0) \int_{a}^{x} \phi(t) \mathrm{d} t .
$$

Here $G(a+0)$ stands for $\lim _{a+} G$, the existence of which follows from the conditions. Note that it is essential that the interval $(a, b]$ contains $b$. A variant not having this requirement is:

If $G:[a, b] \rightarrow R$ is a monotonic (not necessarily decreasing and positive) function and $\phi:[a, b] \rightarrow R$ is an integrable function, then $\exists$ a number $x \in(a, b)$ such that

$$
\int_{a}^{b} G(t) \phi(t) \mathrm{d} t=G(a+0) \int_{a}^{x} \phi(t) \mathrm{d} t+G(b-0) \int_{x}^{b} \phi(t) \mathrm{d} t .
$$

We use the following notations:

$$
\begin{gathered}
\psi(t)=f(x+t)-f(x-t), \\
A_{n, k}=\sum_{r=k}^{n} a_{n, r}, A_{n, 0}=1, \forall n \geq 0, \\
M_{n}(t)=\frac{1}{2 \pi} \sum_{k=0}^{n} \frac{a_{n, k} \cos (k+1 / 2) t}{\sin (t / 2)}, \tau=[1 / t] \text { - the greatest }
\end{gathered}
$$

integer not exceeding of $1 / t$.

Furthermore $C$ will denote an absolute positive constant, not necessarily the same at each occurrence. Throughout this paper, we take $a_{n, k} \geq 0(0 \leq k \leq n)$, and $A_{n, 0}=1 \forall n$.

\section{Main Result}

It is well known that the theory of approximations i.e., tfa, which originated from a well known theorem of Weierstrass, has become an exciting interdisciplinary field of study for the last 130 years. These approximations have assumed important new dimensions due to their wide applications in signal analysis, in general and in digital signal processing [5] in particular, in view of the classical Shannon sampling theorem.

This has motivated by various investigators such as Qureshi ([7,8]), Khan ([4,9]) Chandra [10], Leindler [11] Mishra [5] discussed the degree of approximation of signals (functions) belonging to

$\operatorname{Lip} \alpha, \operatorname{Lip}(\alpha, p), \operatorname{Lip}(\xi(t), p)$ and $W\left(L_{p}, \xi(t)\right)$-classes by using Cesàro and Nörlund means of an infinite series. Qureshi $([12,13])$ have determined the degree of $\tilde{f}(x)$, conjugate of a function $f(x) \in \operatorname{Lip} \alpha$ and $\operatorname{Lip}(\alpha, p)$ by Nörlund means of conjugate series of a Fourier series.

The purpose of this paper is to determine the degree of approximation of $\tilde{f}(x)$, conjugate of a function

$f(x) \in W\left(L_{p}, \xi(t)\right),(p \geq 1)$, by lower triangular matrix means.

We prove:

Theorem 2.1. Let $T \equiv\left(a_{n, k}\right)$ be an infinite regular 
lower triangular matrix such that the elements $\left(a_{n, k}\right)$ be non-negative, non-decreasing with $k \leq n$. If $f: R \rightarrow R$ is a $2 \pi$-periodic, Lebesgue integrable and belonging to the generalized weighted $W\left(L_{p}, \xi(t)\right), p \geq 1$-class, then the degree of approximation of $\tilde{f}(x)$, conjugate of $f(x) \in W\left(L_{p}, \xi(t)\right)$, by lower triangular matrix means $\tilde{\tau}_{n}(f ; x)$ is given by

$$
\left\|\tilde{\tau}_{n}(f ; x)-\tilde{f}(x)\right\|_{p}=O\left(n^{\beta+1 / p} \xi(1 / n)\right) \quad \forall n>0,
$$

provided $\xi(t)$ is positive increasing function of $t$ satisfying the following conditions

$$
\begin{aligned}
& \left\{\int_{0}^{\pi / n}\left(\frac{t|\psi(t)|}{\xi(t)}\right)^{p} \sin ^{\beta p} t \mathrm{~d} t\right\}^{1 / p}=O\left(n^{-1}\right) \\
& \left\{\int_{\pi / n}^{\pi}\left(\frac{\left.t^{-\delta} \mid \psi(t)\right) \mid}{\xi(t)}\right)^{p} \mathrm{~d} t\right\}^{1 / p}=O\left(n^{\delta}\right)
\end{aligned}
$$

and $\quad \frac{\xi(t)}{t}$ is decreasing in $t$

where $\delta$ is an arbitrary number such that $q(1-\delta+\beta)-1>0, q$ the conjugate index of $p$ and conditions (2.2), (2.3) hold uniformly in $x$ and $p^{-1}+q^{-1}=1$.

Note 1. Condition (2.4) implies $\xi(\pi / n) \leq \pi \xi(1 / n)$, for $(\pi / n) \geq(1 / n)$

Note 2. Also for $\beta=0$ our Theorem (2.1) reduces to one of the theorem of Lal and Kushwaha [14].

\section{Lemmas}

In order to prove our Theorem 2.1, we require the following lemma.

Lemma 3.1. Under the conditions of our Theorem 2.1 on $\left(a_{n, k}\right)$, we have

$$
M_{n}(t)=O\left(\frac{A_{n, \tau}}{t}\right), \text { for } \frac{\pi}{n}<t \leq \pi .
$$

Proof. For $\pi n^{-1}<t \leq \pi, \quad(\sin t)^{-1} \leq \pi / 2 t$, for $0<t \leq \pi / 2$, $\tau \leq n$, we have

$$
\begin{aligned}
& \left|M_{n}(t)\right| \\
& =\left|\frac{1}{2 \pi} \sum_{k=0}^{n-\tau-1} a_{n, k} \frac{\cos (k+1 / 2) t}{\sin (t / 2)}+\frac{1}{2 \pi} \sum_{k=n-\tau}^{n} a_{n, k} \frac{\cos (k+1 / 2) t}{\sin (t / 2)}\right| \\
& \leq \frac{1}{2 t}\left|\sum_{k=0}^{n-\tau-1} a_{n, k} \cos (k+1 / 2) t\right|+\frac{1}{2 t}\left|\sum_{k=n-\tau}^{n} a_{n, k} \cos (k+1 / 2) t\right| \\
& \leq \frac{1}{2 t}\left[2 a_{n, n-\tau-1} \max _{0 \leq r \leq n-\tau-1}\left|\sum_{k=0}^{r} \cos (k+1 / 2) t\right|\right. \\
& \left.\quad+\sum_{k=n-\tau}^{n} a_{n, k}|\cos (k+1 / 2) t|\right] \\
& =\frac{1}{2 t}\left[O\left(\frac{a_{n, n-\tau-1}}{t}\right)+A_{n, \tau}\right],
\end{aligned}
$$

and

$$
\begin{aligned}
A_{n, \tau} & =\sum_{k=n-\tau}^{n} a_{n, k} \\
& =a_{n, n-\tau}+a_{n, n-\tau+1}+\cdots+a_{n, n} \\
& \geq a_{n, n-\tau-1}+a_{n, n-\tau-1} \cdots+a_{n, n-\tau-1} \\
& =(\tau+1) a_{n, n-\tau-1} \\
& =\left(\frac{1}{t}+1\right) a_{n, n-\tau-1} \\
& \geq\left(\frac{a_{n, n-\tau-1}}{t}\right)
\end{aligned}
$$

Therefore, $\left|M_{n}(t)\right|=O\left(\frac{A_{n, \tau}}{t}\right) \cdots$ This completes the proof of Lemma 3.1.

\section{Proof of Theorem 2.1}

The $k^{\text {th }}$ partial sum of the conjugate series of the Fourier series (1.2) is given by

$$
\begin{aligned}
& \tilde{s}_{n}(f ; x)= \frac{1}{2 \pi} \int_{0}^{\pi} \cot (t / 2) \psi(t) \mathrm{d} t \\
&+\frac{1}{2 \pi} \int_{0}^{\pi} \frac{\cos (n+1 / 2) t}{\sin (t / 2)} \psi(t) \mathrm{d} t \\
& \tilde{s}_{n}(f ; x)-\left(-\frac{1}{2 \pi} \int_{0}^{\pi} \cot (t / 2) \psi(t) \mathrm{d} t\right) \\
&=\frac{1}{2 \pi} \int_{0}^{\pi} \frac{\cos (n+1 / 2) t}{\sin (t / 2)} \psi(t) \mathrm{d} t
\end{aligned}
$$

Then

$$
\begin{aligned}
& \sum_{k=0}^{n} a_{n, k}\left\{\tilde{s}_{n}(f ; x)-\left(-\frac{1}{2 \pi} \int_{0}^{\pi} \cot (t / 2) \psi(t) \mathrm{d} t\right)\right\} \\
& =\frac{1}{2 \pi} \int_{0}^{\pi}\left(\sum_{k=0}^{n} a_{n, k} \frac{\cos (n+1 / 2) t}{\sin (t / 2)}\right) \psi(t) \mathrm{d} t
\end{aligned}
$$

or,

$$
\tilde{\tau}_{n}(f ; x)-\tilde{f}(x)=I_{1}+I_{2}
$$

Using Riesz-Hölder's inequality, condition (2.2), (2.4), note 1 , the fact that $(\sin t)^{-1} \leq \frac{\pi}{2 t}$, for $0<t \leq \pi / 2$, $p^{-1}+q^{-1}=1$ and the second mean value theorem for integrals, we find 


$$
\begin{aligned}
\left|I_{1}\right| \leq & {\left[\int_{0}^{\pi / n}\left(\frac{t|\psi(t)|}{\xi(t)} \sin ^{\beta} t\right)^{p} \mathrm{~d} t\right]^{1 / p} } \\
& \cdot\left[\int_{0}^{\pi / n}\left\{\frac{\xi(t)}{t \sin ^{\beta} t}\left|M_{n}(t)\right|\right\}^{q} \mathrm{~d} t\right]^{1 / q} \\
\leq & {\left.\left[\int_{0}^{\pi / n} \frac{t|\psi(t)|}{\xi(t)} \sin ^{\beta} t\right)^{p} \mathrm{~d} t\right]^{1 / p} } \\
& \left.\cdot\left[\int_{0}^{\pi / n}\left\{\frac{\xi(t)|\cos (k+1 / 2) t|}{t \sin \beta}\right\}^{q} \mathrm{~d} t\right]^{1 / q}\right]^{1 / 2}(t / 2) \mid \\
= & O\left(\frac{1}{n}\right)\left[\int _ { 0 } ^ { \pi / n } O \left\{\frac{\xi(t)}{\left.\left.t^{2} \sin ^{\beta} t\right\}^{q} \mathrm{~d} t\right]^{1 / q}}\right.\right. \\
= & O\left(\frac{1}{n}\right)\left[\left(\frac{\pi / n}{\sin (\pi / n)}\right)^{\beta q} \int_{h}^{\pi / n} O\left\{\frac{\xi(t)}{t^{2+\beta}}\right\}^{q} \mathrm{~d} t\right]^{1 / q} ; h \rightarrow 0 \\
= & O\left(n^{-1} \xi\left(\frac{1}{n}\right)\right) O\left(n^{2+\beta-1 / q}\right) \\
= & O\left(\frac{1}{n}\right)\left[\int_{h}^{\pi / n}\left\{\frac{\xi(t)}{t^{2+\beta}}\right\}^{q} \mathrm{~d} t\right]^{1 / q} ; h \rightarrow 0 \\
&
\end{aligned}
$$

Now by Riesz-Hölder's inequality, conditions (2.3), (2.4), note 1, Lemma 3.1, the fact that

$$
\begin{aligned}
& (\sin t)^{-1} \leq \frac{\pi}{2 t}, \text { for } 0<t \leq \pi / 2, p^{-1}+q^{-1}=1 \text {, we obtain } \\
& \left|I_{2}\right|=\left|\int_{\pi / n}^{\pi}\right| \psi(t)|| M_{n}(t)|\mathrm{d} t| \\
& \leq\left\{\int_{\pi / n}^{\pi}\left|\frac{t^{-\delta} \psi(t) \sin ^{\beta} t}{\xi(t)}\right|^{p} \mathrm{~d} t\right\}^{1 / p}\left\{\int_{\pi / n}^{\pi} \mid \frac{\left.\left.\xi(t) M_{n}(t)\right|^{q} \mathrm{t} t\right\}^{-\delta} \sin ^{\beta} t}{1 / q}\right. \\
& =\left\{\int_{\pi / n}^{\pi}\left(\frac{t^{-\delta}|\psi(t)|\left|\sin ^{\beta} t\right|}{\xi(t)}\right)^{p} \mathrm{~d} t\right\} \\
& \quad \cdot\left\{\int_{\pi / n}^{\pi}\left(\frac{\xi(t)}{t^{-\delta} \sin ^{\beta} t} O\left(\frac{A_{n, \tau}}{t}\right)\right\}^{q} \mathrm{~d} t\right\} \\
& =O\left(n^{\delta}\right)\left\{\int_{\pi / n}^{\pi}\left(\frac{\xi(t) A_{n, \tau}}{t^{-\delta+\beta+1}}\right)^{q} \mathrm{~d} t\right\}^{1 / q}
\end{aligned}
$$

Since $A$ has non-negative entries and row sums one,

$$
\begin{aligned}
& =O\left(n^{\delta}\right)\left\{\int_{1 / \pi}^{\pi / n}\left(\frac{\xi(1 / y)}{y^{\delta-\beta-1}}\right)^{q} \frac{\mathrm{d} y}{y^{2}}\right\}^{1 / q} \\
& =O\left(n^{\delta} \frac{\xi(\pi / n)}{\pi / n}\right)\left\{\int_{1 / \pi}^{\pi / n}\left(\frac{\mathrm{d} y}{y^{\delta q-\beta q+2}}\right)\right\}^{1 / q} \\
& =O\left(n^{\delta+1} \xi\left(\frac{1}{n}\right)\right)\left\{\frac{\left(\frac{n}{\pi}\right)^{q(-\delta+\beta)-1}-\left(\frac{1}{\pi}\right)^{q(-\delta+\beta)-1}}{q(-\delta+\beta)-1}\right\}^{1 / q} \\
& =O\left(n^{\delta+1} \xi\left(\frac{1}{n}\right)\right) O\left(n^{-\delta+\beta-1 / q}\right) \\
& =O\left(n^{\beta+1-1 / q} \xi(1 / n)\right)=O\left(n^{\beta+1 / p} \xi(1 / n)\right)
\end{aligned}
$$

Combining $I_{1}$ and $I_{2}$ yields

$$
\left|\tilde{\tau}_{n}(f ; x)-\tilde{f}(x)\right|=O\left(n^{\beta+1 / p} \xi(1 / n)\right) .
$$

Now, using the $L_{p}$-norm, we get

$$
\begin{aligned}
\left\|\tilde{\tau}_{n}(f ; x)-\tilde{f}(x)\right\|_{p} & =\left\{\int_{0}^{2 \pi}\left|\tilde{\tau}_{n}(f ; x)-\tilde{f}(x)\right|^{p} \mathrm{~d} x\right\}^{1 / p} \\
& =O\left\{\int_{0}^{2 \pi}\left(n^{\beta+1 / p} \xi(1 / n)^{p} \mathrm{~d} x\right\}^{1 / p}\right. \\
& =O\left\{n^{\beta+1 / p} \xi(1 / n)\left(\int_{0}^{2 \pi} \mathrm{d} x\right)^{1 / p}\right\} \\
& =O\left(n^{\beta+1 / p} \xi(1 / n)\right) .
\end{aligned}
$$

This completes the proof of our Theorem 2.1.

\section{Applications}

The following corollaries can be derived from our Theorem 2.1 .

Corollary 5.1. If $\beta=0$ and $\xi(t)=t^{\alpha}, 0<\alpha \leq 1$, then the generalized weighted class $W\left(L_{p}, \xi(t)\right)$ reduces to class $\operatorname{Lip}(\alpha, p)$ and the degree of approximation of a function $f(x) \in \operatorname{Lip}(\alpha, p)$ is given by $\left\|\tilde{\tau}_{n}(f ; x)-\tilde{f}(x)\right\|_{p}=O\left(n^{-\alpha+1 / p}\right)$.

Proof of corollary 5.1. From our Theorem 2.1 for $\beta=0$, we have

$$
\begin{aligned}
& \left\|\tilde{\tau}_{n}(f ; x)-\tilde{f}(x)\right\|_{p}=\left(\int_{0}^{2 \pi}\left|\tilde{\tau}_{n}(f ; x)-\tilde{f}(x)\right|^{p} \mathrm{~d} x\right)^{1 / p} \\
& =O\left(n^{1 / p} \xi(1 / n)\right)=O\left(\frac{1}{n^{\alpha-1 / p}}\right), p \geq 1 .
\end{aligned}
$$

This completes the proof of corollary 5.1.

Corollary 5.2. If $p \rightarrow \infty$ in corollary 5.1, then for 
$0<\alpha<1,\left\|\tilde{\tau}_{n}(f ; x)-\tilde{f}(x)\right\|_{p}=O\left(n^{-\alpha}\right)$.

Corollary 5.3. If $a_{n, k}=p_{n-k} P_{n}^{-1}, P_{n} \neq 0, \xi(t)=t^{\alpha}$ then the degree of approximation of $\tilde{f}(x)$, conjugate of $f \in \operatorname{Lip}(\alpha, p)$ by Nörlund means

$\tilde{\tau}_{n}(f ; x)=P_{n}^{-1} \sum_{k=0}^{n} p_{n-k} \tilde{s}_{k}(f ; x)$ of the conjugate series of Fourier series is given by

$$
\left\|\tilde{\tau}_{n}(f ; x)-\tilde{f}(x)\right\|_{p}=O\left(n^{-\alpha+1 / p}\right) .
$$

\section{Corollary 5.4. If}

$a_{n, k}=p_{n-k} P_{n}^{-1}, P_{n} \neq 0, \xi(t)=t^{\alpha}, p \rightarrow \infty$ then the degree of approximation of $\tilde{f}(x)$, conjugate of

$f \in \operatorname{Lip} \alpha$ by Nörlund means $\tilde{\tau}_{n}=P_{n}^{-1} \sum_{k=0}^{n} p_{n-k} \tilde{s}_{k}$ of the conjugate series of Fourier series is given by

$$
\left\|\tilde{\tau}_{n}-\tilde{f}\right\|_{\infty}=\left\{\begin{array}{l}
O\left((n+1)^{-\alpha}\right), 0<\alpha<1, \\
O(\log (n+1) \pi e /(n+1)), \alpha=1 .
\end{array}\right.
$$

Corollary 5.5. If $a_{n, k}=p_{n-k} q_{k} R_{n}^{-1}$ such that $R_{n}=\sum_{k=0}^{n} p_{n-k} q_{k} \neq 0, R_{y} y^{-\alpha}$ is monotonic non-decreasing then the degree of approximation of $\tilde{f}(x)$, conjugate of a function $f \in \operatorname{Lip} \alpha$, by generalized Nörlund means $\tilde{\tau}_{n}(f ; x)=R_{n}^{-1} \sum_{k=0}^{n} p_{n-k} q_{k} \tilde{s}_{k}(f ; x)$ of the conjugate series (1.2) satisfies equation (5.1).

\section{Remarks}

Remark 6.1. Lal and Kushwaha [14]. The degree of approximation $\left\|\tilde{\tau}_{n}(f ; x)-\tilde{f}(x)\right\|_{p}=O\left(n^{-\alpha+1 / p}\right)$. determined by Qureshi [13, p. 561, L. 12] tends to $\infty$ if $0<\alpha<3^{-1}<1$ and $p=2$ and also for other values. Therefore, this deficiency has encouraged to investigate degree of approximation of conjugate of functions belonging to $\operatorname{Lip}(\alpha, p)$ considering $p^{-1}<\alpha<1$.

\section{Acknowledgements}

The authors are grateful to his beloved parents for their encouragement to his work. The authors are grateful to the referee for his valuable suggestions and useful comments for the improvement of this paper. The authors are also thankful to the AM Editor in chief Prof. Chris Cannings, University of Sheffield, UK and AM Editorial Assistant Ms. Tian Huang, Scientific Research Publishing, USA for their kind cooperation during communication.

\section{References}

[1] A. Zygmund, "Trigonometric Series Vol. I," Cambridge University Press, Cambridge, 1959.
[2] O. Töeplitz, "Über Allgemeine Lineare Mittelbildungen," Prace Matematyczno-Fizyczne Journal, Vol. 22, 1913, pp. 113-119.

http://www.zentralblatt-math.org/zmath/en/journals/searc $\mathrm{h} / \mathrm{?a}=00003590$

[3] L. McFadden, "Absolute Nörlund Summability," Duke Mathematical Journal, Vol. 9, 1942, pp. 168-207. doi:10.1215/S0012-7094-42-00913-X

[4] H. H. Khan, "On the Degree of Approximation to a Function Belonging to Weighted $\left(L^{P}, \xi(t)\right)$ Class," Aligarh Bulletin of Mathematics, Vol. 3-4, 1973-1974, pp. 83-88.

[5] V. N. Mishra, "Some Problems on Approximations of Functions in Banach Spaces," Ph.D. Thesis, Indian Institute of Technology, Roorkee, 2007.

[6] V. N. Mishra, "On the Degree of Approximation of Signals (Functions) Belonging to the Weighted $W\left(L_{P}, \xi(t)\right.$ ), $(p \geq 1)$-Class by Almost Matrix Summability Method of Its Conjugate Fourier Series," International Journal of Applied Mathematics and Mechanics, Vol. 5, No. 7, 2009, pp. 16-27.

[7] K. Qureshi, "On the Degree of Approximation of a Function Belonging to Lipa," Indian Journal of Pure and Applied Mathematics, Vol. 13, No. 8, 1982, pp. 898-903.

[8] K. Qureshi, "On the Degree of Approximation of a Function Belonging to the Class Lip $(\alpha, p)$," Indian Journal of Pure and Applied Mathematics, Vol. 13, No. 4, 1982, pp. 466-470.

[9] H. H. Khan, "On the Degree of Approximation to a Function by Triangular Matrix of Its Conjugate Fourier Series II," Indian Journal of Pure and Applied Mathematics, Vol. 6, 1975, pp. 1473-1478.

[10] P. Chandra, "Trigonometric Approximation of Functions in $L_{P}$-Norm," Journal of Mathematical Analysis and Applications, Vol. 275, 2002, pp. 13-26. doi:10.1016/S0022-247X(02)00211-1

[11] L. Leindler, "Trigonometric Approximation in $L_{P}$-Norm," Journal of Mathematical Analysis and Applications, Vol. 302, 2005, pp. 129-136. doi:10.1016/j.jmaa.2004.07.049

[12] K. Qureshi, "On the Degree of Approximation of Conjugate of Function Belonging to the Lipschitz Class by Means of Conjugate Series," Indian Journal of Pure and Applied Mathematics, Vol. 12, No. 9, 1981, pp. 11201123.

[13] K. Qureshi, "On the Degree of Approximation of Conjugate of Function Belonging to the Class $\operatorname{Lip}(\alpha, p)$ by Means of Conjugate Series," Indian Journal of Pure and Applied Mathematics, Vol. 13, No. 5, 1982, pp. 560-563.

[14] S. Lal, and J. K. Kushwaha, "Approximation of Conjugate of Functions Belonging to the Generalized Lipschitz class by Lower Triangular Matrix Means," International Journal of Mathematical Analysis, Vol. 3, No. 21, 2009, pp. 1031-1041. 